\title{
Towards the Design and Evaluation of Interactive Social Technologies: A Survey Report
}

\author{
Souvik Mukherjee \\ Department of Computer Science \\ Lakehead University \\ Thunder Bay, Ontario \\ smukher2@lakeheadu.ca
}

\author{
Ngudup Tsering \\ Department of Computer Science \\ Lakehead University \\ Thunder Bay, Canada \\ ntsering@lakeheadu.ca
}

\author{
Jinan Fiaidhi \\ Department of Computer Science \\ Lakehead University \\ Thunder Bay, Canada \\ jfiaidhi@lakeheadu.ca
}

\begin{abstract}
With new technologies, increasing mechanization and automation, almost all challenging social and economic issues that people face can be investigated towards a prediction or a solution. Digital technologies have connected the world in a single knot, but the technical knowhow does not contribute to solving these challenges. Encapsulation of technological advancement and social responsibility is highly needed. This calls for cross-regiment and inter-field solutions coupled with social enthusiasm. This survey provides a study on two aspects of social relations within a connected world. It also investigates and reviews solutions and work for advanced interactive technology. The survey draws on major insights from few researchers highlighted in our reference list.
\end{abstract}

Keywords-computer-mediated communication, global virtual team, social media engagement

\section{INTRODUCTION}

Computer science and its diverse application arenas address the most urgent and complex problems across the globe, thus finding its way in the day-to-day lives of modern society. Technology has found its epitome in almost all spheres of human lives and the society as a whole, and yet mere knowledge about information technology and automation sciences fail to address the social aspect of any venture that technology undertakes to do. Hence, a crossdisciplinary application deployment is desired to address the parallel social problems along with the technological needs.

Humans have come a long way in technology. Community of practice is now sharing information digitally with variety of technologies, but there is still a yearning for having the true "society" presence of a "connected" world. Our reliance on technology more than physical beings may be costing us social interactions and ultimately a community building. Let us discuss two scenarios that automation and AI revolution has put us into. As the initial step, man programs machines on how to learn. Then the scenario is branched. The first branch has the machines to augment man's intelligence, and its subsequent deployment as a support system in the achievement of technological goals. This situation is commonly known as the "man-andmachine" situation. The second branch has the machines run into competition with man for rigorous development to suit man's needs and their subsequent deployment to serve humans. But machines become more intelligent in the process surpasses human intelligence. This situation is known to be "man-vs-machine" situation. We in the modern day have experienced the occurrences of both these phenomena in our daily lives. While the first situation of collaborative work helps build on a better society, the second situation might sound as an adverse effect on community building. Thus, the focus on the social aspect of technology has become more and more desirable as we dig deeper into automation. As the Nobel Prize winner Christian Lous Lange puts it, "technology is a useful servant, but a dangerous master."

Major issues that impede the blossoming of creativity, innovation, and excellence are the unconducive environs, be it while collaborating with a team overseas, or collaborating with strangers over interactive reality games. The goal of this report is to study, review, explore, analyze and infer on the various aspects of interactive technologies and automation to identify prevalent issues, and offer plausible and feasible solutions. This survey provides a study on two aspects of social relations within a connected world. It includes a study that look into the "linguistic and cultural barriers in globally distributed virtual work teams (GVTs), and loneliness and isolation in an increasingly connected world"2. These two issues are deemed as global social causes worthy of the attention of technology, and may be addressed via the deployment of interactive technologies.

Three aspects of human emotions form the basic pillars of efficient social interaction, collaboration, and communication. These are as follows.

- Feedback - refers to the transmission of evaluative or corrective information about an action or process source in a social interaction environment.

- Self-reflection - indicates the capacity to exercise introspection and to attempt to learn more about their fundamental nature and essence based on feedback.

- Behaviour change - refers to any transformation or modification of human behaviour based on selfreflection, and subsequent attitude change.

These are basic tools that help monitor social interaction and involvement. As can be seen from their very definitions, these aspects form a chain rule of processes that mediate effective intergroup communication.

According to Dr. Helen He "The aspects of social perspective is drawn from the basic ideologies of feedback, self-reflection, and behavior change" 3 , and subsequent development of interactive technologies for efficient intergroup communication. Interactive technologies are

\footnotetext{
${ }^{1}$ https://www.nobelprize.org/prizes/peace/1921/lange/lecture/

${ }^{2} \mathrm{https}: / /$ web.cs.dal.ca/ research/Seminars(2018-19).htm\#he

${ }^{3}$ https://web.cs.dal.ca/ research/Seminars(2018-19).htm\#he
} 
influenced by social collaboration and community building which connect strangers at public places ${ }^{4}$, subsequently driving effective communication, and bridging all prejudiced gaps.

The rest of the report is organized as follows. The immediate section that follows speaks of a reference that almost all works of collaborative work have considered, and is a book that addresses the problems of the present and future of automation, and remedies potential solutions. It also speaks of the hierarchy of needs and consequent discussion of the employment of technologies towards the social good aspect. The section ends by discussing the ill effects of the hierarchy on society throwing light on controversial and challenging theories like that of Universal Basic Income, and the Purpose Problem as a potential social impact of it. The next section talks about the social challenges being addressed to, in the report, and surveys works that have been conducted in those fields. They include discussion on GVTs and associated culture and linguistics, and the direct proportion of connection with loneliness and isolation. The next section discusses about the application of the pillars of effective social interaction towards the development of one of the most influential social psychology theories, known as the Intergroup Contact Theory, its problems, and its reformulation towards reshaping a collaborative community. The report then moves to explore interactive technologies, and reviews and recommends collaborative mixed reality as a plausible and feasible solution to address the social challenges faced by technology [2]. The penultimate section discusses and concludes the qualitative case study, and the final section discusses the challenges, and the scope for future work in this emerging area of technology for society.

\section{REFERENCE THEORIES}

\section{A. 21 Lessions for the $21^{\text {st }}$ Century}

" 21 lessons for the $21^{\text {st }}$ century is a book written by social psychologist Yuval Noah Harari in 2018. After his previous publications "Sapiens looked deep into humankind's past and Homo Deus considered" [31] the scenario of existing in a future powered by intelligent design, this book seeks to focus on the biggest questions on the present day global agenda, cutting through the information overwhelm of the virtual world.

This book comprises of 21 chapters that asks 21 questions. These questions build on ideas to take the pulse of current global climate, entangling political, technological, social and existential queries highlighting how daily lives of humans are impacted by them. Overall, Harari poses a fascinating and controversial big-picture on the queries about the future of humanity owing to the automation and technological intervention. The information provided ranges from disruptive technologies that compete for our attention, the prospective and limitations of automation, advances in infotech and biotech, ethics of automation, equality and liberty in the age of big data, the future of education systems, resilience skills, the importance of self-awareness,

${ }^{4}$ Helen $\mathrm{Ai}$ He. Loneliness and isolation in an increasingly digital world. Humane Tech meetup, June 2018. https://helenaihe.com/research/connection/ self-knowledge, and meditation in an age when machines surpass humans in knowing themselves.

Thus, this book derives from the basic emotions of feedback, self-reflection, and behaviour change in an era of automation which are also the pillars of effective intergroup interaction. The book also takes on the social aspect of technologies, which is the purpose of this report that addresses the social challenges, and brings about recommendations for collaboration and communication. Thus, this book has been referenced by scientists from fields of HCI, CSCW, and UbiComp worldwide.

\section{B. Maslow's Hierarchy of Needs}

This is a motivational psychological theory of human development and how humans partake on behavioural motivation presented by A.H. Maslow book in 1943 [30]. It also summarizes on how effort and motivation are correlated. It shows a five-tier model of human needs, often depicted as hierarchical levels within a pyramid. Needs in the lower strata ought to be satisfied before individuals can attend to higher needs.

\section{1) The Pyramid Model [30]}

The model categorized the needs as follows.

- Physiological needs

- Safety needs

- Belongingness and love needs

- Esteem needs

- Self-actualization needs

\section{2) Need Identification}

The model can be broken down into deficiency needs which comprise of the bottom four needs, and growth needs which comprise of the topmost need (self-actualization). Deficiency needs are derived from deprivation from fulfillment of the needs, and motivation is said to be inversely proportional to the fulfillment of these needs. After fulfillment, these needs become salient needs. Growth needs however are derived from self-fulfillment, and motivation is directly proportion to the needs being met. Growth needs thus come not from deficiency but from desire for personal growth. These levels are often seen to be continuously overlapping with each other as shown in [30].

To summarize, the hierarchy of needs deems selfactualization to be the highest form of social need and thus signifies the need for the social aspect to be the most valued need once the basic technological needs are met and the immediate requirements are addressed. And within humans, self-reflection and subsequent behaviour change can be viewed as the self-actualization process.

\section{Universal Basic Income}

In a survey by Futurism entitled "Universal Basic Income (UBI)" many questions has been raised including the Robot-to-Worker increase ratio and many debatable social issues related to technology which has been studied and explored by many countries around the world as an answer to the existence of human population and fulfillment of their needs is at question due to technological advancements, putting jobs at stake that are vulnerable to 
automation $^{5}$. UBI is defined as a fixed amount, at a level sufficient for subsistence, given by the state to all its citizens regardless of income or work status.

Actually, the UBI satisfies the lower levels of basic needs in the hierarchy of needs and not the higher psychological and self-fulfillment needs. When UBI comes into picture, that is with automation and a consequent lack of jobs, needs for belongingness and esteem is not satisfied. And since these levels are left unattended in the hierarchy, reaching the self-actualization stage becomes almost impossible. Thus, with the advancement of technologies the idea for having a social impact should be kept parallel to ensure effective and efficient social community building with interaction and collaboration among various groups.

\section{The "Purpose Problem"}

The "purpose problem" discussed by Bill Gates ${ }^{6}$ to redress the potential social impacts of UBI, and subsequent discussion of the social aspects of technologies as the sole purpose that shall remain once all other purposes are met as mentioned in another book by Yuval Harari [32]. Also has been echoed by others ${ }^{7}$.This is in alliance with the growth needs, which implies that the desire for self-fulfillment would increase with attainment of the same. This further consolidates the fact that self-reflection arising from feedback, and behaviour and attitude change are essential social building grounds for effective social collaboration.

The purpose problem was discussed by gates after referencing the book Homo Deus by Yuval Harari. His take on the hierarchy of needs is a little bit different than the explanation of UBI. Gates considers the situation when all the requirements have been met by technology, that is when people have surpassed the deficiency needs, and are in the growth phase. Dr. He argues that $\mathrm{AI}^{8}$ taking over the world is an engineering problem, but the purpose problem is more like a control problem, that is, once the needs are met and all the purpose is served, what would people do? The answer to this obviously is the self-fulfillment, which would eradicate the lack of purpose, and would continue to benefit society as it grows within each individual.

These theories have been referenced and researched extensively towards mitigating the social dilemmas via the process feedback, self-reflection, and behaviour change as prompted in the abstract of the research seminar hosted by Helen Ai He [2,29,34], where the two social dilemmas are discussed. All these discussions bring us to the same inference, that the social aspect of technological advancement is parallel in development and deployment. Also, feedback, self-reflection, and behaviour change are essential components crucial for the design of interactive technologies for benefiting collaborative communication.

\footnotetext{
${ }^{5}$ https://futurism.com/images/universal-basic-income-answerautomation

${ }^{6}$ https://www.gatesnotes.com/Books/Homo-Deus

${ }^{7}$ https://helenaihe.com/2018/03/22/universal-basic-incomehuman-needs-what-we-gain-and-what-we-may-lose/

8

https://helenaihe.com/2018/11/25/future-of-humanity/
}

\section{VIRTUAL TEAMS INQURY}

Many organisations aim to diversify their workforce by focusing on attracting and retaining employees from diverse cultural backgrounds and nationalities. A multicultural workforce can bring advantages, such as a wider range of viewpoints and a greater opportunity to compete on the global market. The differences in culture and language can impede effective communication. Research shows that workers who are not fluent in the primary language in the workplace can find it difficult to communicate their needs or respond to requests [29]. Such challenges aggravate many folds when body language cannot be read in Virtual Teams [20].

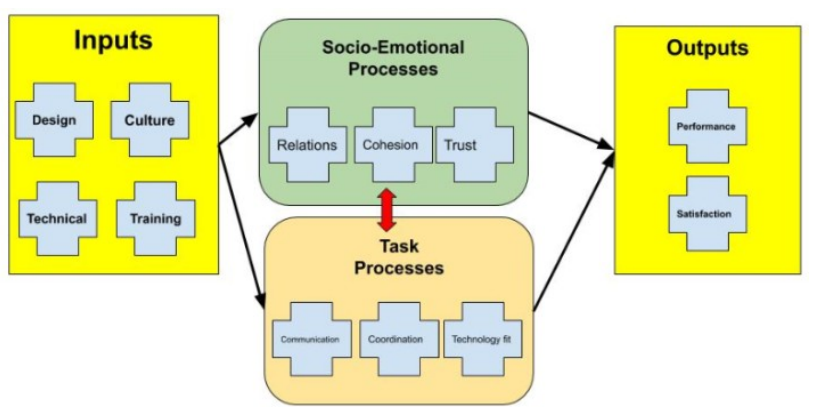

Fig 1: Virtual Team Research adapted from [20].

\section{A. Global Virtual Teams}

With the rise of globalization and the explosion of technology, we have witnessed striking transformations in the workplace. Technologies have influenced society through its products and processes and have influenced the quality of life, and guided the ways people act and interact. This also gave rise to the concept of GVTs which is becoming popular and will probably become mainstream in the coming decades with increasing acceptance of ideas like 'work from home' and rapid spread of pandemic diseases such as COVID-19. However, GVTs are heavily dependent on Computer-Mediated Communication (CMC) tools [18]. Therefore, a structured interaction is extremely crucial to have a successful GVT.

The structure of GVTs as shown in Figure 4 can be broadly classified into four parts: input, socio-emotional processes, task processes, and output [19]. Input modules focus on structuring the interactions, establishing the shared norms, creating a clear team structure. One of the critical aspects of the input module is the design and update of the shared knowledge database. Social-emotional processes recognize emotional problems and provide mitigation tactics to achieve cohesion and trust among team members ${ }^{9}$. The primary challenge here is how people can solve the trust problem in a short time. One of the answers could be the swift trust paradigm as it suggests that team members assume from the beginning that the other team members are trustworthy, and adjust that assumption during the lifetime of the team [20]. Task processes are actions that team members carry out to manage and realize their project, or collective goal. The most important components are communication and coordination.

\footnotetext{
${ }^{9}$ https://en.m.wikipedia.org/wiki/Virtual_team
} 
When properly managed, GVTs have higher potential to outperform the traditional teams, as it comprises cultural diversity that affects group decision making [21]. Different backgrounds and experiences in the group members encourage creativity, at the same time conflicting viewpoints explore multiple options securing many facets. One major merit of GVTs is equality in the workplace [22]. As the team is virtual, the discrimination on the basis of age, race, disability and gender is far less prominent. However, there are challenges that GVTs encounter for the same reasons.

\section{B. Cultural Challenges}

"Cultural diversity in GVTs has an innate lack of shared mental models which can lead to ambiguity in communication" [21, 23]. While some cultures prefer indirect communication, some opt direct. These levels of formality vary from culture to culture and therefore extremely difficult to gauge [34]. Same can be said about the range of emotional expression and perception of time.

\section{Linguistic Challenges}

The linguistic challenges of GVTs can be attributed to the fact that $\mathrm{CMC}$ tools reduce access to the social and contextual signs and therefore increases the miscommunications. For the non-native speaker of the lingua franca, there is considerable cognitive load that can potentially affect their performance which can lead to biased impression and attribution errors [18]. Biased impression and attribution errors will also impact trust and cohesion negatively [24]. Furthermore, sparse transitive memory in GVTs on account of a low frequency of communication can reduce the information about individual work context (who knows what in the team) and is often not transferred to new members.

\section{Survey Findings on Virtual Teams}

\section{1) Society of Human Resource Management: Virtual teams}

This survey was published in 2012 and found that almost one-half of organizations (46\%) used virtual teams in their workplace ${ }^{10}$ even as early as 2012 with an obvious finding that organizations with multinational operations were more than twice as likely (66\%) to use virtual teams compared with organizations with US based operations (28\%) [25]. Organizations with multinational operations collaborates with teams distributed across geographical locations with virtual teams as traditional methods fall short to meet the ever-changing needs and growth. Within the organizations that use virtual teams, the fundamental reason for the adoption was contributed to the inclusion of talent in different geographic locations with 53\%, followed closely by collaboration boosting from different geographical locations and improving productivity with $49 \%$ and $39 \%$ respectively. Furthermore, successful teamwork behaviour for a virtual team was attributed to brainstorming solutions for problems with $72 \%$, setting goals with $68 \%$, and developing plans for team initiatives with $63 \%$. In addition,

$10 \mathrm{https} / /$ www.slideshare.net/shrm/virtual-teams-final it showed that the primary challenges faced by the virtual team were associated with trust and relationship building (51\%), time differences (49\%), distribution of work (32\%), differences in cultural norms $(26 \%)$.

\section{2) RW3 Culture Wizard ${ }^{11}$}

This survey was published in 2018, and surveyed 1,620 respondents from 90 countries [26]. The survey is inclusive and diverse that can reflect the effect of the language barrier and cultural diversity in a virtual team. The first difference that was noticeable in this survey was the overwhelming majority of respondents ( $89 \%)$ were members of at least one virtual team which is almost double of that from 2012. The steep rise in GVTs adoption has many contributing factors, but the primary cursor is the exponential rise in technology and globalization. The distribution was discernible with $62 \%$ of respondents on one to three virtual teams and $27 \%$ on at least four teams. As multicultural issues play a role in nearly all GVTs, the survey explored the complexity of these issues. In the findings, $89 \%$ of their virtual teams typically include at least two cultures, and more than onethird of them consist of four or more cultures indicating that single culture dominance is quickly getting out of trend. The survey further shed a light on the severity of various cultural challenges in GVTs.

The regretful findings from these surveys are that the challenges of GVTs eight years ago are still persistent and relevant with no big strides of technological updates fixing them. Even though the appeal for GVT is palpable, it is hard to get it right. The guidelines by Harvard Business Review: Getting Virtual Teams Right published 2014 is intuitive and implementable [27]. Their recommendations revolve around four points: the Right Team, the Right Leadership, the Right Technology, and finally the Right Touchpoint.

\section{E. Plausible Design Solutions}

\section{1) Automated Sensing Technologies}

Impression sensing technology has increasingly evolved with advancement of modern technologies. It is more accessible and can be easily deployed. Such characteristics propel this choice to forefront in detecting behaviours for multiparty meetings. As both verbal and nonverbal behaviours contribute to establishment of impression, it is prudent to explore this area further [28].

As we found in the many research conducted in this regard, the existing sensing technologies are more geared towards the verbal aspects and the feedback thereof. Providing only quantitative feedback on the verbal aspects of the communication can often be inimical to the teams and organisations where members are from different cultural and linguistic backgrounds, ergo designing a technology that takes into consideration: which behavioral signs to capture; how are they expressed; and what are their interpretations is

\footnotetext{
${ }^{11}$ https://content.ebulletins.com/hubfs/C1/Culture\%20Wizard/LL2018\%20Trends\%20in\%20Global\%20VTs\%20Draft\%2012\%20and\%20a $\%$ 20half.pdf
} 
vital. Such a feedback channel can contribute positively in providing clear impressions of one another.

\section{2) Exploration different channels of Impression} Construction

The impression of competence is one of the important areas of evaluation employed in many organisations. As the impression construction is subjective, a true picture of competence cannot be drawn with CMC that is available at present. More often than not, the existing system favours more on communication strength than the task and other core skill strengths. Such biased design in systems can be detrimental to the organization, yet no particular traction is seen in this area. In multicultural and multi-linguistic milieu, a lingua-franca of communication for the native speaker is advantageous in establishing positive impression, but the non-native speaker has a very limited control in such circumstances, and it's not reflective of core competence. In order to mitigate such issues alternative channels for impression construction is necessary. Alternative impressions can be constructed by indulging them in different parameters of analysis. Especially in multicultural and multi-linguistic environments, collaboration and trust are in short supply, and the determination of intercultural competence among members is crucial. A shared selfreflection questionnaire used by Dr. He et al [28], though rudimentary, provided some level of insight during communication and served as a meta-channel to communication. Similar approach with extensive and psychologically sound self-reflection parameters can be used to learn and gauge the dynamics of communication, and continuous feedback in the form of several and simple interpretations should be provided. These self-reflection parameters will have to cover a broad spectrum of different cultural cues and general personality traits.

\section{3) Learning Intercultural Competence}

The need for intercultural competence is accentuated by its inclusion in the ACTFL Guidelines, United States and the Common European Framework for Languages, Europe. Culture-specific learning and exchange is far lacking and should be supplemented by culture-general learning in the era of globalization. Such a step will stem the ability of an individual to communicate effectively with people from different cultures in various circumstances. In a multicultural and multi-linguistic organization, members from different backgrounds have to exchange ideas and communicate effectively in order to succeed [34]. Lacking such profound training can result in loss of important assets, and can prove ruinous and expensive. Tools to support people in developing intercultural competence are essential while mitigating perceptions of intercultural conflict [29].

\section{THE Dillamma of CONNECTED WORLD VS ISOLATION}

"Human beings are a social species and thus have an innate longing for connection",12, that is, they crave and seek social connections [3]. But in the modern "socially connected" world, with the advent and globalization of social media people tend to be connected virtually, yet lonely when it comes to physical or mental connections [4]. Research shows that "we would rather text, than talk". This implies our inclination towards reliance on technology and subsequent connection with others is more than reliance on each other [9]. As a result, "we are alone, together." And the fact that humans are tending towards more social connections without actually socializing is giving rise to loneliness and isolation in this increasingly connected world [1].

Isolation is defined as the seclusion of an individual from any or all commodities and access, basically resulting from separation. Here we refer to social isolation, which essentially means suffering from a social exclusion and separation of an individual from the rest of the society. Loneliness can be seen as an adverse human emotional response to isolation. This is typically a social limitation and usually includes unpleasant feelings about a lack of connection or communication with the social world. Thus human separation can be viewed as a reason resulting from loss of social connection - what humans are hard-wired for.

\section{A. Role of Social Media}

The advent, development, and very design of social media can be viewed as a direct or a point cause for this emotional and social phenomenon. The engagement of our lives to social media connects everyone virtually but increases the physical communication gap manifold [10], leading us to feel even more isolated and lonely while indulging in constant comparison and competition with what we see and hear around us posing a greater risk for mortality [8]. The design of these technologies is regarded as "disruptive" and occupies a large portion of our brain's limited cognitive resources. Furthermore, social media development is evolving in a channelized and attentionbiased route. Thus, our attention towards being more social physically and thus subdues loneliness and isolation ${ }^{13}$ is somehow being lost inside virtual social media connections [2]. Thus, people are losing themselves somewhere due to loss of social communications and connections, inability to be creative (such as doing deep work), and no critical reflection towards collaborative work.

\section{B. Health Impacts}

Loneliness and isolation are on a global surge, with negative impacts on health owing to an emotional breakdown from the connected community [7]. And these health adversities have greater impact than other health regulating factors like obesity and smoking [6]. UK has a quarter of all ages who feel emotionally unconnected to others. In the USA in $2014,12 \%$ to $23 \%$ people had no one to confide in, as compared to the $8 \%$ in 1985 . In the EU, approximately $38 \%$ suffer from intense loneliness. Loneliness affects physical and mental health as evident from a few meta-analysis of studies on loneliness [5]. The figures from this meta-analysis say that odds of dying early increases by around $45 \%$ when a person is left in loneliness, as opposed to only 5\% from air pollution, $20 \%$ from

\footnotetext{
${ }^{13} \mathrm{https}: / /$ helenaihe.com/research/connection/
}

${ }^{12} \mathrm{https}: / /$ helenaihe.com/research/connection/ 
obesity, and 30\% from drinking and smoking. Loneliness and isolation are thus currently a global public health risk.

\section{Design of Prototypes}

To address this mushrooming social issue, there has been extensive and promising development and deployment of interactive technology prototypes and subsequent release of these tools [11]. All these technologies have been built on grounds of $\mathrm{HCI}, \mathrm{CSCW}$, and UbiComp, and stand on four pillars of social aspects which drive communication and interaction. They are: feedback, self-reflection, and behaviour change as the three basic ideologies, and strangers as the fourth one. Strangers were added as the fourth pillar of addressing social issues and developing of prototypes for the same much later. The initial interactive technologies to remedy social isolation and loneliness were based only on the former three, and typically connected families and friends together, that is, peer-to-peer interaction and communication.

\section{1) Isolated Groups}

The idea behind the development of peer-to-peer interactive technologies and tools was that isolation results from the lack of social contacts which typically fall under the group of peers. And this has been seen leading to aloneness, despair, helplessness, and subsequently to other unpleasant effects, including increased mortality. The groups most prone to being affected by these scenarios were reviewed to be children and senior citizens. These social groups were the first targets of the prototypes to be designed. Isolated groups in children might include children with disorders, children with chronic diseases, children with different mindset and outlook, or children at day care centers. The socially isolated senior groups might comprise of home dwelling seniors suffering from chronic pains. Both subjects may be seen as analogous and complementary. Thus the recommended peer-to-peer prototypes to address them serve two targeted social groups with a single purpose.

\section{2) Motivation}

The interest in technology for social connectivity and efficient communication was prevalent as early as digital portraits, and photo frames. It was also sparked on social communication devices that were typically home-based. Technological development in this field has been closely idolized from the concept and design of "communication appliances for intimate social networks"[34]. Although most prototypes have focused on visual communication, the concreteness of audio communication is still valued more. Furthermore, synchronous messaging has been seen to attract socially isolated groups more than asynchronous messaging [12]. The term synchronous messaging refers to a mode of communication in which the sender desires quick response to his/her messages, while asynchronous messaging refers to the setting of a message queue which might not require immediate answering by the recipient. In all current interaction networks, a combination of the two is used, sometimes even integrating video communications.

A grounded theory approach is necessary for the design and analysis of each successive deployment and engagement with target users. For the purpose of gathering information for prototyping a combination of observations, interviews, focus groups and diary studies prove vital. For isolated groups or isolated individuals, participating in social interaction might be difficult in real-time owing to their physical or mental situations and conditions, and thus asynchronous communication approach is adopted while designing the following prototypes, resulting from interview studies.

\section{3) Families in Touch Prototype}

The first experimental prototype being discussed and reviewed is known as Families in Touch (FIT) prototype, and derives its name from a sense of "touch" that it provides via interfaced asynchronous communication. The interface is very similar to digital photo frames with asynchronous audio-visual messaging capability for tactile interaction. Families or peers might receive a notification when the isolated individual touches the frame indicating a potential connection, thus directly remedying loneliness. But this prototype has no personal messaging system, that is, it notifies through smart messages rather than typed messages.

\section{4) Ringo prototype}

For delving deeper into the issue of isolation and loneliness and development of better prototypes, a diary study is essential, which summarizes and determines the situations in which these individuals actually had the communication they desired, and when they had a desire for social contact, but the process could not be completed. Based on the insights derived from this study another prototype may be developed which was initially given the name Ringo. This is actually an android based system, more or less like a tab and supported both personal message and media sending capabilities. It is very similar to common messenger applications available nowadays, with a smart replying interface.

Interview studies from several diverse isolated groups in different settings prove the fact that not always technology has been essential or efficient in dealing with isolation. This is because existing technologies does not take into full account this social aspect of media communications, that is, social media might not actually connect people and mediate interactions.

\section{5) Implications for Design}

There are thus several design implications that need to be considered while designing interactive technologies to reduce loneliness and isolation in this increasingly connected world [12]. They are as follows:

- Avoid common computing aesthetics and conventions.

- $\quad$ Support self expressions

- Leverage family pictures to encourage engagement.

- Do not break social ties with existing relationships.

- Respect existing applications of devices and communication patterns.

- Emphasize asynchronous communication but provide room for synchronous communication too.

- Provide multiple media coverage (that is, audio, video, personal messages, and the like).

- Use tactile interaction techniques. 
- Do not be linguistically biased.

- Build assistive technology

- Support sufficient privacy, security, and reliability.

\section{6) InTouch Prototype}

These implications have been deployed in the design of a prototype named InTouch, to engage, encourage, and enhance peer-to-peer communication. The interface is almost similar to modern messenger applications with a more closed target group. It has both synchronous and asynchronous messaging capabilities, and also supports almost all media. The pre-set messages are still present, along with the capability to send a "wave". It gives users the simulation of a photo frame along with emailed notification capabilities.

\section{INTERGROUP CONTACT THEORY}

Dr. Helen Ai He et al [2] proposes the implication and application of intergroup contact theory ${ }^{14}$ as a prospective towards addressing the loneliness and isolation, as well as mitigating cultural barriers.The three pillars towards mediating effective interaction and communication between different social, cultural, and linguistic groups are discussed earlier, which include feedback, self, reflection, and behaviour change. The fourth pillar however, has not been discussed - strangers. "Connecting strangers in public places pave way for mitigating both the social dilemmas, as well as the incorporation of feedback, self-reflection, and behaviour change within the environment in action." 15 . Strangers form a pillar because humans tend to be polarized towards peers, but are completely unknown to strangers. Hence the question of bias does not arise and helps in seamless development of interactive technologies. Public places have been chosen as the area of application and employment of these social technologies to address the issues of loneliness, isolation, and cultural and linguistic barriers among strangers. This is simply because gathering drives communication. All this together, form the basis of the formulation of a social psychology theory known as Intergroup Contact Theory.

\section{A. The Theory and Optimal Conditions}

Intergroup Contact Theory is one of the most influential and challenging theories on reducing prejudice, subsequent bias, and thus leading to better contact among groups. It was proposed by Gordon Allport in 1954 [33]. As feedback, self-reflection, behaviour change, and strangers in public places for the basic social aspects of interaction and communication, the intergroup contact theory suggested four optimal contact conditions that was proposed to be essential for effective intergroup communication. They are:

- Equity between

- Common focused

- Between group members cooperation

- Authority Support from authorities

\section{B. Empirical Evidences}

There are many empirical evidences to support this theory, both early and recent researches [13, 14]. Evidences showed negative impacts in communication when all four conditions were violated. Studies from interdependencies developed on ships and maritime union investigated the optimal conditions, and provided integrity of the intergroup contact theory [14]. Similar findings from Philadelphia police among other studies ${ }^{16}$. Studies from public housing in New York City and Newark provided robust evidences [14]. The housing projects were chosen such that there were racially desegregated housings in New York, while segregated ones in Newark. "Allport's formulation continues to receive support across a variety of situations, groups, and societies. Recent empirical evidence deems the four key conditions as crucial. Investigations conducted in situations that do not provide key conditions leads to adverse effects" [14].

A handful of studies also report positive contact effects even when the optimal conditions were not in the situation [13]. Also, the intergroup contact theory can be applied to a broader spectrum of fields and not only confined to ethnic groups. Studies of intergroup contact theory have also taken into account contact groups such as children, seniors, homosexuals, mentally and physically challenged, victims of chronic disease, and even computer programmers. These varied investigations broaden the employment arenas of the theory, and subsequent contradictions pertaining to the application arenas.

\section{Basic Problems with the initial contact theory}

There thus exists some basic problems with the original intergroup contact theory that might not have been assessed or addressed [13]. These are described below.

- The casual sequence problem

- Independent variable specification problem

- Unspecified process of change problem

- The generalization of effects problem

\section{Processes that operate through Intergroup Contact}

Four interrelated processes change through intergroup contact and mediate feedback, self-reflection, and behaviour change [13]. They are listed below.

- Ingroup reappraisal

- Learning about the outgroup

- Changing behaviour

- Generating effective ties

\section{E. Intergroup Contact Theory Restructuring}

These considerations pave ways for the restructuring of the intergroup contact theory [12]. The four processes involved in the optimal conditions of the intergroup contact theory may overlap and interact in complex ways to mediate intergroup contact, which in turn is channelized by long-term close acquaintanceship. Constructive contact relates more closely towards the development of crossgroup friendships. Thus, friendship here is regarded as a

16

https://www.annualreviews.org/doi/10.1146/annurev.psych.49.1.65 
fifth optimal condition of the intergroup contact theory for effective intergroup communication and interaction.

\section{F. Meta-analysis of reformulated contact literature}

An extensive meta-analytic of the reformulated contact theory was carried out in 2006 with 713 samples from 515 studies, that surveys and evaluates the optimal conditions of the reformulated intergroup contact theory [13]. The findings of this meta-analysis validate the reformulated theory, suggesting that more intergroup contact is generally associated with lower levels of intergroup discrimination. One main finding of the meta-analysis was that all these optimal conditions act as "facilitating" conditions and not as "essential" conditions towards achieving effective intergroup interaction. Thus, optimal conditions are not necessary requirements, but when present, act as catalysts or facilitators that enhance the tendency for positive contact experience. Intergroup contact not only improves the feedback and behaviour to the immediate outgroup members involved in the interaction, but that of the entire outgroup. The results also generalize to other types of social isolation between diverse groups.

\section{DESIGN RECOMMENDATIONS}

So far, we have looked at task specific design recommendations towards seamless interactive communication among virtual teams, and isolated groups. But we need to highlight "common humanity" and thus interactive technologies that prompt spontaneous and meaningful connections among people. We have reviewed what is known as "Humane Design", which essentially aims at realigning technology with humanity's social interests. The objective of these designs is to give an intuition of the fact that strangers stand as the fourth pillar of flawless communication since teams or groups with known members and peers are subject to polarization and emotional bias, which is viewed as a constraint in diversity. Virtual public places like GVTs [2] are chosen because gathering drives communication. This encapsulation is what shall rejuvenate a disconnected world of strangers.

We draw major insights from the work of Helen $\mathrm{Ai} \mathrm{He}$ et al [2], and move forward in the same lines to recommend the use of interactive technologies to revive the sense of connection at the same time serving the purpose of communication and problem solving, that is, interactive technologies should address to the technical issues as well as the linked social challenges prevalent in any community. We also view mixed reality coupled with collaboration as a probable and plausible solution towards the design of interactive technologies, and move on to explain the need for collaborative mixed reality, and its subsequent deployment in the design of collaborative mixed reality games as a remedy to remove both cultural and linguistic barriers, and loneliness and isolation. We look into collaborative mixed reality gaming from the perspective of feedback, self-reflection, and behaviour change as being the three pillars which center the revolution of mitigating social dilemma. Thus Dr. He recommends the use of HCI, CSCW, and UbiComp research towards the design and evaluation of collaborative mixed reality based applications for social $\operatorname{good}^{17}$, and put forth inspirations and examples from mixed reality games and its prototyping in collaboration and communication as benchmarks.

\section{A. Mixed Reality (MR)}

The ability of MR based systems to engage and interact with both physical and virtual objects and environments gives it a huge number of potential applications. Currently $\mathrm{MR}$ is being deployed as prototypes and commercial products and services in the fields of education, engineering, healthcare, entertainment, etc. Some classic commercial examples of MR based systems include Microsoft Hololens and Hololens 2, and Magic Leap.

\section{B. Collaborative Mixed Reality}

The potential social aspect of MR based systems have not yet been discovered or deployed towards the interaction goal between virtual teams and isolated groups, and there is little or no work in this field in areas of HCI, CSCW and UbiComp [17]. And here is where collaborative mixed reality comes into play [14]. The intuition behind this is "shared experiences in mixed reality emphasizes on target scenarios for collaboration", as explained by commercial MR based application systems. Collaborative MR upholds the interaction goal of connecting strangers in virtual or public places with the help of MR.

MR interfaces have the capability of overlaying multimedia interactions. This can subsequently be deployed to enhance communication regardless of proximity, that is, connections and interfaces that go "beyond being there". But there has been very little work on collaborative MR towards HCI and CSCW, and this is what we urge to do. There are five key advantages of collaborative MR environments as described by [14].

\section{Collaborative Mixed Reality Games}

Collaborative mixed reality games are basically gaming interfaces based on collaborative MR, which enable shared social experiences and learn cultural competence. In this, players interact with the physical and virtual environments and with each other in real-time [15]. These are social interaction procedures that enable different modes of interaction in which players engage with combinations of co-located physical environments as in intergroup communication, or remote virtual environments as in GVTs. The rewards of these collaborative MR games traces back to the self-fulfillment strata of the pyramidal hierarchy, and help develop on feedbacks through the games, selfreflection through the feedbacks and rewards, and finally a behaviour change and subsequent attitude change towards participants of the game. This same theory may be applied to strangers in public places like ingroups and outgroups, and can also be employed to virtual environments like GVTs to reduce cultural and linguistic barriers.

\section{Inspirations}

Some of the technological inspirations include the following.

\footnotetext{
${ }^{17}$ https://web.cs.dal.ca/ research/Seminars(2018-19).htm\#he
} 
- Actiwait - a street crossing pong game in Germany, by Urban Invention.

- $\quad$ Small World Machine - an interactive MR based game to rejuvenate ties between India and Pakistan, an initiative by Coca Cola.

- Piano Staircase - a musical staircase for interaction among strangers in Sweden brought about by Volkswagen, and many others.

\section{CHALLENGES AND SCOPE FOR FUTURE WORK}

While MR based collaborative interfaces can be built for seamless communication, and offer a plausible and feasible solution to address the challenges commonly faced among social groups in virtual forums and public places [16], there are some limitations to the commercial deployment of these interfaces, which is why this technology is still in prototyping stage, and not many researchers are talking about it.

To start with, these sophisticated collaborative systems are not easy to build [15]. They require considerable effort for designing the virtual elements, choosing appropriate technologies, defining boundaries, and ensuring safety and security. This is the primary reason why these type of projects are typically commercial with a dedicated team of designers and developers, and not a personal research project. A major design challenge posed is the combination of technologies, which might include integration of a lot of hardware and software components in an efficient way in order to enable flawless experience. This too is a drawback as it makes these interfaces both difficult to build and hard to maintain [16]. Another major issue with these collaborative MR devices might be their design, development, installation, and maintenance cost. Expenses might see a surge, when wanting to deploy it in a large scale. But when employed on a commercial scale in the form of an industrial project, this method proves to be perfect for addressing the social needs of the present and the future from the perspective of collaboration and communication.

As a scope of future work thus, we would be trying to actually go ahead and make a collaborative application interface for addressing the prevalent social issues with the help of industrial support for building, deploying, and maintaining the applications at the same time providing users with a seamless communication experience, serving the purpose of interaction goal.

\section{CONCLUSION}

This report first discusses the shortcomings of the technological aspects of modern day computer intervention in the daily lives of humans, and addresses to the social aspect of the same through the design of interactive technologies. It performs a qualitative case study of quantitative surveys with respect to the most common social challenges when it comes to intergroup communication. The social challenges include cultural and linguistic barriers in widely deploying virtual groups and reducing secluded in an increasingly connected world. Some related theories has been surveyed, along with the pillars of efficient and effective interaction. All these are further deployed in the argument that collaborative mixed reality might be the plausible and feasible solution to the social aspect of emerging technologies, and subsequently address to the social challenges posed. Collaborative mixed reality games are proposed as an area of exploration pertaining to the engagement and enhancement of effective intergroup communication, and serve the interaction goal of connecting strangers in public places. Finally the challenges in their design, development, deployment, and maintenance are discussed, and this gives an insight as to why only commercial prototypes have been produced so far. But the prospective future work points towards a direction of possible collaborative work in the fields of $\mathrm{HCI}, \mathrm{CSCW}$, and UbiComp, and open a new area of socio-technical research.

\section{ACKNOWLEDGEMENTS}

This is a revised preprint of a survey essay submitted to Dr. J. Fiaidhi.

\section{REFERENCES}

[1] M. Young, The Technical Writer's Handbook. Mill Valley, CA: University Science, 1989.

[2] Helen Ai He. 2018. "Transcending Boundaries: Applying Intergroup Contact Theory to the Design of Collaborative Mixed-Reality Games". Position paper at the Hybrid Events Workshop at ACM CSCW 2018.

[3] Cacioppo, John T., and William Patrick. Loneliness: Human nature and the need for social connection. WW Norton \& Company,c2008.

[4] Brown, Brené. Daring greatly: How the courage to be vulnerable transforms the way we live, love, parent, and lead. Penguin, 2015.

[5] Pinker, Susan. The village effect: How face-to-face contact can make us healthier and happier. Vintage Books Canada, 2015

[6] Yan, Zhimin, Xun Yang, Lei Wang, Yuhan Zhao, and Lin Yu. "Social change and birth cohort increase in loneliness among Chinese older adults: a cross-temporal meta-analysis, 1995-2011." International psychogeriatrics 26, no. 11 (2014): 1773-1781

[7] Hawkley, Louise C., and John T. Cacioppo. "Loneliness matters: a theoretical and empirical review of consequences and mechanisms." Annals of behavioral medicine 40.2 (2010): 218-227.

[8] Holt-Lunstad, Julianne, Timothy B. Smith, Mark Baker, Tyler Harris, and David Stephenson. "Loneliness and social isolation as risk factors for mortality: a meta-analytic review." Perspectives on Psychological Science 10, no. 2 (2015): 227-237.

[9] Chua, Trudy Hui, and Leanne Chang. "Follow me and like my beautiful selfies: Singapore teenage girls' engagement in selfpresentation and peer comparison on social media." Computers in Human Behavior 55 (2016): 190-197.

[10] Turkle, Sherry. Alone together: Why we expect more from technology and less from each other. Hachette UK, 2017.

[11] Vogel, Erin A., Jason P. Rose, Lindsay R. Roberts, and Katheryn Eckles. "Social comparison, social media, and self-esteem." Psychology of Popular Media Culture 3, no. 4 (2014): 206.

[12] R. Baecker, K. Sellen, S. Crosskey, V. Boscart, and Barbara B. Neves. "Technology to reduce Social Isolation and Loneliness." 2014

[13] Thomas F. Pettigrew. "Intergroup Contact Theory." 1998.

[14] Thomas F. Pettigrew. "A Meta-Analytic Test of Intergroup Contact Theory." 2006.

[15] Mark Billinghurst and Hirokazu Kato. "Collaborative Mixed Reality." 1999.

[16] Sultan A. Alharthi. "Collaborative Mixed Reality Games.” 2018.

[17] William Green, Boris de Ruyter. "The design and evaluation of interactive systems with perceived social intelligence: five challenges." Dec, 2009.

[18] Nikolaos Avouris, Christos Sintoris, Christina Katsini. "Humancomputer interaction for social good: the case of digital government evaluation and redesign project."

[19] Pamela Hinds and Sara Kiesler. 2002. Distributed Work. MIT Press. 
[20] Powell, Piccoli and Ives (2004) p.8, Anne Powell, Gabriele Piccoli, and Blake Ives. Virtual teams: a review of current literature and directions for future research. The DATA BASE for Advances in Information Systems - Winter Vol. 35, issue 1, 2004.

[21] Jarvenpaa and Leidner, (1999) p.794, Sirkka Jarvenpaa and Dorothy E. Leidner, Communication and Trust in Global Virtual Teams, Organization Science; Special Issue: Communication Processes for Virtual Organizations, Vol. 10, issue 6, 1999, p. 791-815.

[22] Shachaf, Pnina. "Cultural diversity and information and communication technology affects global virtual teams: An exploratory study." Information \& Management (2008): 131-142. November 11, 2014.

[23] Bergiel, Blaise J, Erich B. Bergiel and Phillip W. Balsmeier. "Nature of virtual teams: a summary of their advantages and disadvantages." Management Research News 31.2 (2008): 99-110. November 14, 2014.

[24] Hertel, Guido; Geiser, Susanne; Konradt, Udo (2005). "Managing virtual teams: A review of current empirical research". Human Resource Management Review. 15: 69-95. doi:10.1016/j.hrmr.2005.01.002.

[25] D. Sandy Staples and Lina Zhao. 2006. The Effects of Cultural Diversity in Virtual Teams Versus Face-to-Face Teams. Group Decis. Negot. 15, 4 (July 2006), 389-406. DOI:https://doi.org/10.1007/s10726-006-9042-x

[26] Society for Human Resource Management (SHRM) Survey Findings: Virtual Teams, 2012.
[27] CultureWizard: Trends in High-Performing Global Virtual Teams, 2018

[28] Harvard Business Review: Getting Virtual Teams Right, 2014

[29] Helen Ai He, Naomi Yamashita, Ari Hautasaari, Xun Cao, and Elaine M. Huang. "Why Did They Do That? Exploring Attribution Mismatches Between Native and Non-Native Speakers Using Videoconferencing." In Proceedings of the 2017 ACM Conference on Computer Supported Cooperative Work and Social Computing (CSCW 2017), pp. 297-309. ACM, 2017.

[30] Maslow, A. H. (1943). "A theory of human motivation". Psychological Review, 50(4), 370396. https://doi.org/10.1037/h0054346

[31] Yuval Noah Harari. "21 lessons for the $21^{\text {st }}$ century”. Spiegel \& Grau, Jonathan Cape. August, 2018.

[32] Yuval Noah Harari. "Homo Deus: a brief history of tomorrow". Harvill Secker. September, 2016.

[33] Allport, G. W. The nature of prejudice. Cambridge, MA: Perseus Books. 1954.

[34] Helen Ai He, Naomi Yamashita, Chat Wacharamanotham, Andrea B. Horn, Jenny Schmid, Elaine M. Huang. "Two Sides to Every Story: Mitigating Intercultural Conflict through Automated Feedback and Shared Self-Reflections in Global Virtual Teams". In Proceedings of the ACM: Human Computer Interaction (PACM 2017), 1(2), pp. 51 72. ACM, 2017 